\title{
Indexical and Referential Pointing in Chimpanzees (Pan troglodytes)
}

\author{
David A. Leavens \\ University of Georgia and \\ Yerkes Regional Primate Research Center, \\ Emory University
}

\author{
William D. Hopkins \\ Berry College and \\ Yerkes Regional Primate Research Center, \\ Emory University
}

\author{
Kim A. Bard \\ Yerkes Regional Primate Research Center, \\ Emory University
}

\begin{abstract}
The spontaneous index finger and other referential pointing in 3 adult, laboratory chimpanzees (Pan troglodytes) who have not received language training is reported. Of 256 total observed points, 254 were emitted in the presence of a human to objects in the environment; therefore, the points were communicative. Indicators of intentional communication used by the subjects included attention-getting behaviors, gaze alternation, and persistence until reward. Thus, pointing by these chimpanzees was intentionally communicative. These data imply that perspective-taking and referential communication are generalized hominoid traits, given appropriate eliciting contexts. Index finger pointing was more frequent with the subjects' dominant hands. This study refutes claims that indexical or referential pointing is species-unique to humans or dependent on linguistic competence or explicit training.
\end{abstract}

This study was initiated when a chimpanzee began to regularly point to accidentally dropped food items outside his home cage and beyond his reach in the presence of an experimenter. We sought to determine if this pointing behavior could be defined as intentional communication, and not simple reaching, by comparing the pointing behavior of this subject and two cagemates with pointing and intentional communication in human infants.

The production and comprehension of referential pointing in preverbal humans have been increasingly studied in recent decades with respect to their significance for the attribution of intentionality in preverbal children (e.g., Bates, Camaioni, \& Volterra, 1975; Desrochers, Morissette, \& Ricard, 1995; Fogel \& Thelen, 1987; Leung \& Rheingold, 1981). Functionally, a point directs the attention of an

David A. Leavens, Department of Psychology, University of Georgia, and Yerkes Regional Primate Research Center, Emory University; William D. Hopkins, Department of Psychology, Berry College, and Yerkes Regional Primate Research Center, Emory University; Kim A. Bard, Yerkes Regional Primate Research Center, Emory University.

This research was supported in part by National Institutes of Health Grants RR-00165, RR-01658, RR-03591, and NS-29574. The Yerkes Regional Primate Research center is fully accredited by the American Association of Laboratory Animal Care. David A. Leavens gratefully acknowledges the partial support of a Delyte and Dorothy Morris Doctoral Fellowship from Southern Illinois University at Carbondale and a University-Wide Graduate School Assistantship from the University of Georgia. We thank Charles Hyatt for his participation in this project and Kelly McDonald for valuable assistance in coding the videotapes. We thank Cobie Brinkman, Alan Fogel, Dorothy Fragaszy, and H. Lyn Miles for helpful comments and discussion.

Correspondence concerning this article should be addressed to David A. Leavens, Department of Psychology, University of Georgia, Athens, Georgia 30602. observer to an object or location in the immediate environment. From a structural standpoint, pointing in humans may involve an extended index finger with full arm extension or may be as subtle as a slight raise of the chin and a glance. Following Blake, O'Rourke, and Borzellino (1994), we argue that "the essence of pointing lies in its function, which is referential communication, and not in mere index finger extension" (p. 202). We focus here on pointing with extended fingers to distal objects, with the putative goal of directing the attention of an observer to those distal objects. Given (a) a signaler, (b) an observer, and (c) a salient distal stimulus, the communicative intent of a point is inferred from attention-getting behavior (e.g., vocalizations), arm and finger extensions to the distal objects, and the monitoring with gaze of the attentional status of an observer (Bates et al., 1975; Leung \& Rheingold, 1981). Intentional communication is distinguished from intentional behavior by the use of social agents as a means to achieve some end (see Bard, 1990 and 1992, for discussion).

As noted by Tomasello and Call (1994), "Many apes that have had extensive contact with humans... learn to use referential pointing to direct the attention of humans to distal entities and locations. This has been well documented for all four great ape species" (p. 287). Nevertheless, a number of authorities have recently claimed either that apes do not point (Butterworth \& Grover, 1988; Corballis, 1991; Donald, 1991) or that apes do not point with the index finger (Povinelli \& Davis, 1994). For example, Donald (1991) remarked that the putative lack of intentionality in the communicative gestures of home-reared chimpanzees is "emphasized by the absence of spontaneous pointing behavior" (p. 127). With respect to indexical pointing, Povinelli and Davis (1994) asserted that, in laboratory-reared chimpanzees that exhibit pointlike gestures, "pointing with the 
index finger does not develop, even in those subjects trained to respond to human indexical pointing" (p. 134).

Other researchers have observed that laboratory-reared chimpanzees exhibit referential pointing to direct the attention of conspecifics or humans to objects or locations in the environment (Boysen \& Berntson, 1989; Boysen, Berntson, Shreyer, \& Hannan, 1995; Fouts, Hirsch, \& Fouts, 1982; Krause, Bodamer, \& Fouts, 1995; Povinelli, Nelson, \& Boysen, 1990; Savage-Rumbaugh, 1984, 1986; Woodruff \& Premack, 1979). Referential pointing has also been observed in all the other great ape species, including bonobos (Savage-Rumbaugh, 1984, 1986), gorillas (Patterson, 1978), and orangutans (Call \& Tomasello, 1994; Miles, 1990). However, although pointing in language-naive chimpanzees has been incidentally reported in previous studies, no systematic observations of referential pointing per se have been reported in language-naive chimpanzees without overt training to point. Furthermore, no previous study compared the incidence of points by chimpanzees in the presence and absence of human beings (see Call \& Tomasello, 1994, for audience effects on pointing in two orangutans). Therefore, despite the widespread reports of pointing behavior in chimpanzees, until the communicative nature of these gestures is established, inferences about the intentionality of the gestures can be reasonably debated. For example, in the human developmental literature, the transition from pointing in an exploratory fashion-and without reference to adult attention-to pointing with reference to adult attention (i.e., gaze monitoring of the adult, presumably to assess the adult's attentional status, or attentiongetting vocalizations, or both) is taken to be evidence of the advent of intentional communication in human preverbal children, usually beginning at about 12 months of age (e.g., Bates et al., 1975). Finally, previous studies or observations of pointing in apes have failed to describe the pointing gestures in structural terms; this makes comparisons across studies exceedingly difficult.

The research reported here was conducted to answer three questions. First, Is the pointing behavior exhibited by our chimpanzee subjects communicative? If so, we hypothesized that the incidence of pointing in the presence of human observers would be significantly greater than in the absence of human observers. Second, Is the behavior intentional? We hypothesized that if the behavior is intentional, the incidence of chimpanzees' use of gaze alternation between the objects of the points and the human observers would be significantly greater than gazes directed only to the distal objects or only to the observers. Gaze alternation and attention-getting behaviors are the standard indicators of intentional communication in human children (e.g., Bates et al., 1975; Desrochers et al., 1995; Leung \& Rheingold, 1981) and in apes (Bard, 1990; Plooij, 1978; Tomasello, Call, Nagell, Olguin, \& Carpenter, 1994). Third, What are the structural characteristics of the points exhibited by our chimpanzee subjects? We distinguish between pointing with a single digit and whole-hand pointing (corresponding in form to Fogel \& Hannan's, 1985, "SPREAD," where all fingers are abducted, with the additional proviso that the fingers be simultaneously extended and directed at an iden- tifiable object in the environment). For descriptive purposes, we divided single-digit pointing into two categories, indexical and all others, and identified which hand was used to point.

\section{Method}

\section{Subjects}

The primary subject of this study was Clint, a 14-year-old, nursery-reared male chimpanzee (Pan troglodytes) housed at the Yerkes Regional Primate Research Center of Emory University. Clint is a subject in an ongoing study of laterality and cognition and has been extensively tested with an automated, joystickmediated apparatus referred to as the Language Research Center Computerized Test System (LRC-CTS; described in Washburn \& Rumbaugh, 1992). The other 2 subjects were females: Anna, 39 years old, and Flora, 53 years old. Both Anna and Flora were housed with Clint at the time of this study. Anna's early rearing history is unknown (possibly wild-caught), and Flora was nurseryreared. None of these subjects has been trained in any languagelike communication system. Furthermore, none of the subjects has been overtly trained to point.

\section{Procedure}

Throughout this study (July 19 to August 19, 1994), Clint was tested on a matching-to-sample task with the LRC-CTS (Washburn, Hopkins, \& Rumbaugh, 1989). Correct responses resulted in the delivery of food rewards (peanuts or seedless grapes) from a rotary turnstile feeder to a polyvinyl chloride (PVC) tube connected to the cage mesh. These rewards usually fell through the PVC tube to the floor of the cage at the subject's right. The experimenter usually operated two such units simultaneously, resulting in frequent absences to attend to the other unit, which was out of sight and hearing in another part of the great ape housing facility.

Occasionally, a peanut or grape dispensed for a correct response would overshoot the PVC pipe and land on the floor in the corridor outside the cage mesh, beyond the reach of the subject. Less often, a reward would be correctly delivered to the PVC tube but would then bounce off either the cage mesh or the subject's hand to land on the floor outside the cage mesh and outside the subject's reach. The misdelivery of food was an entirely random event and not engineered by the experimenters. Prior to the onset of this study, the experimenter would typically pick up any fallen food and deliver it to Clint-pointing was not, at any time previous to this study, observed or required by the experimenter as a condition for the delivery of a misdelivered food item. Early in July 1994, however, as David A. Leavens returned to tend the LRC-CTS, Clint was observed to repetitively vocalize, point with his index finger, and alternate his gaze between a fallen grape and the experimenter. At this point, data collection began.

A videocamera was placed in the corridor outside Clint's home cage and adjusted to capture his behavior and the unpredictable misdelivery of rewards. The videotaped sessions ranged from 48 to $93 \mathrm{~min}(M=73 \mathrm{~min})$. Because Clint was the only subject being tested on the LRC-CTS, the videocamera was positioned to capture Clint's head movements and gaze behavior. Therefore, during the vast majority of points emitted by the females, who did not operate the LRC-CTS, their heads were outside the field of view of the videocamera; hence their gaze behavior could not be observed.

A total of over 18 hours of videotape, comprising 15 test 
sessions, was coded in real time by two observers. The two states recorded were (a) observer present and (b) observer absent, to measure audience effects. Points were defined as single-digit or whole-hand extensions directed to an object outside the cage mesh. When a point occurred in real time, the videotape was rewound and replayed as many times as necessary, at both normal and slow speed, to record the onset and offset of the point to the nearest second (to measure durations of the points), the pointing subject's name, hand use (left, right, or both hands), digit use (which digit was extended), vocalizations (present or absent) or other attentiongetting behavior, the putative objects of the points (food or nonfood), the number of looks directed to the object of the point (to assess gaze behavior), and the number of looks directed at the experimenter (or other human observer-also to assess gaze behavior). Comments on any other notable information in the context or form of each point were made on the data sheets. The time codes were elapsed times from the beginnings of the sessions and were obtained from a chronometer recorded directly onto the videotape at the time of original recording. The latencies from the onset of points to the delivery of food rewards, the overall probability of reward, and the durations of points as a function of whether the points were followed by a reward were calculated for each subject.

\section{Reliability}

Fifteen test sessions were conducted and videotaped (total time was $1,038 \mathrm{~min}$ ). Two independent observers were used to score the videotapes. The primary observer coded 11 sessions; the secondary observer (who was naive to the specific hypotheses being tested) coded 10 sessions, with 1 session used for training of the secondary observer. Thus, there were 5 sessions jointly coded that served to establish reliability. Interobserver agreement on whether a point occurred was high at $99.5 \%$ (Cohen's $\kappa=.842$; agreement that a point did not occur was calculated on 5-s intervals). Given that the two observers agreed that a point occurred, further reliability estimates were calculated on gaze (81.5\%; Cohen's $\kappa=.55$ ), vocalization $(84.1 \%$; Cohen's $\kappa=.52)$, presence of observer (100\%), and digit use (85.7\%; Cohen's $\kappa=.80$ ). Cohen's kappa corrects for agreement by chance alone: Kappas above .40 are considered fair; above .60, good; and above .75, excellent (Bakeman \& Gottman, 1986).

Initially, one observer recorded all finger extensions as points, and the other distinguished points from probes (ends of digits touching an object, usually the end of the PVC food delivery tube; this behavior corresponds in form to what Blake et al., 1994, called a "poke" for human children, and the majority of tagging behaviors described by Boysen et al., 1995, for a chimpanzee). Thus, during tabulation of all points recorded on the original data sheets, 8 finger protrusions originally recorded as points were reclassified as probes and 11 additional protrusions were discarded because of ambiguity or a lack of consensus by the researchers. All 8 probes were emitted in the absence of a human observer, and 6 of the 11 ambiguous cases were emitted in the absence of a human observer.

\section{Results}

\section{Observer Presence}

We recorded 175 points from Clint, 53 from Anna, and 28 from Flora, for a total of 256 points over all 3 subjects. Of these 256 points, 254 were exhibited in the presence of a human observer (99\%), and only two were exhibited in the absence of humans $(1.0 \%), \chi^{2}(2, N=256)=248.00$, $p<.001$.

\section{Gaze and Attention-Getting Behavior}

The camera was positioned to capture Clint's gaze behavior during pointing (see the Method section); very little data on gaze behavior from Anna and Flora were recorded; therefore, only Clint's gaze behavior could be included for this analysis. During 8 of Clint's 175 points, either his head was occluded or he had moved his head out of the field of view-these 8 points were also omitted from the present analysis, leaving 167 points with information about gaze behavior.

As shown in Table 1, Clint exhibited significantly more gaze alternations while pointing than predicted by chance, $\chi^{2}(2, N=167)=154.64, p<.001$. Gaze alternation, attention-getting vocalizations, or both occurred during $80 \%$ of Clint's points. Seventy-six percent of Clint's points were accompanied by gaze alternation, and $24 \%$ were accompanied by vocalizations. Expected frequency was calculated with the following reasoning: Since in the course of pointing, Clint could (a) alternate his gaze between a distal object and the observer, (b) look fixedly either in the direction of his point or at the observer, or (c) look elsewhere, expected frequencies were 167 divided by 3 for each of three cells, or 55.67.

Beginning during the first recording session, both of Clint's cagemates, Anna and Flora, were observed to point with their index fingers, after banging with the dorsal portions of their wrists against the cage mesh (i.e., they exhibited attention-getting behavior), to the experimenter's lower left lab coat pocket in which he always carried peanuts. Of Anna's 53 points, 26 (49\%) were immediately preceded by cage-banging, vocalizing, or both, whereas of Flora's 28 points, $4(14 \%)$ were immediately preceded by cagebanging and none was accompanied by vocalizations. Anna's corpus was analyzed for bouts of pointing, in which a bout was defined as a series of at least two points with interpoint intervals of less than $5 \mathrm{~s}$. Nine such bouts were identified (mean number of points $=2.67$; range $=2-4$ points per bout), 7 of which included cage-banging or vocalizing at least once $(78 \%)$. Flora exhibited 4 bouts (mean number of points $=2.25$; range $=2-3$ points per bout), one of which included cage-banging (25\%). Thus,

Table 1

Gaze and Vocalizations Produced During Points by Clint

\begin{tabular}{|c|c|c|c|c|c|c|}
\hline \multirow[b]{2}{*}{ Gaze behavior } & \multicolumn{2}{|c|}{$\begin{array}{c}\text { With } \\
\text { vocalizations }\end{array}$} & \multicolumn{2}{|c|}{$\begin{array}{c}\text { Without } \\
\text { vocalizations }\end{array}$} & \multicolumn{2}{|c|}{ Total } \\
\hline & $n$ & $\%$ & $n$ & $\%$ & $n$ & $\%$ \\
\hline $\begin{array}{l}\text { Alternation } \\
\text { Look at object or }\end{array}$ & 34 & 20 & 94 & 56 & 128 & 7 \\
\hline $\begin{array}{l}\text { exp } \\
\text { onl }\end{array}$ & 6 & 4 & 33 & 20 & 39 & 2 \\
\hline Look anywhere else & 0 & 0 & 0 & 0 & 0 & \\
\hline Total & 40 & 24 & 127 & 76 & 167 & 10 \\
\hline
\end{tabular}


Anna engaged in more attention-getting behavior and exhibited more points than Flora.

\section{Effects of Reinforcement}

Given a point, was there an association between reinforcement and whether another point was emitted within 60 $s$ of the original point? To assess the effects of reward on the probability of subsequent pointing, given that one point had already been emitted, the presence or absence of additional points for an arbitrary 60-s interval after either food reinforcement or no food reinforcement was recorded. The subjects exhibited additional points significantly more often after no reinforcement was given than after receipt of a food reward, $\chi^{2}(1, N=246)=15.12, p<.001$.

The mean durations of points in seconds were $4.9(S D=$ 4.7), $1.3(S D=.9)$, and 1.5 $(S D=.9)$ for Clint, Anna, and Flora, respectively. The correlation between duration of a point and the latency of food reinforcement was statistically significant only for Clint, Pearson's $r=.323, p=.002$, $n=90$. The probabilities of food reward after the emission of a point were $.514, .321$, and .214 for Clint, Anna, and Flora, respectively; the reciprocals of these probabilities may be construed post hoc as the variable ratio reinforcement schedules for the subjects.

In general, points that were followed by food rewards ceased prior to receipt of the food rewards. For all three subjects, the difference between (a) the latencies from the onsets of the points to the delivery of food rewards and (b) the durations of the points was used to calculate a mean time of termination of a point relative to food delivery of $-5.92 \mathrm{~s}(S D=9.97 ; n=113$ total rewards). In contrast, points were maintained through the experimenters' verbal acknowledgments of the points: Mean termination of a point relative to the verbal acknowledgments was $2.56 \mathrm{~s}$ ( $S D=$ 5.349; $n=148$ ).

\section{Generalization to Others}

Most of the points exhibited by our subjects were emitted in the presence of Leavens $(n=236)$; however, evidence of generalization was found, as 20 points were made in the presence of seven other individuals with Leavens absent. Clint emitted four points in the presence of William D. Hopkins, six points in the presence of another researcher, three points in the presence of each of three different primate care technicians, and one point in the presence of a veterinary technician. Of the 14 points generalized by Clint to other humans, 11 were accompanied by gaze alternation (79\%) and 6 of these 11 by vocalizations (55\%). During one of the remaining three generalized points, Clint's gaze and vocalization behavior could not be classified because his head was occluded by the veterinary technician. Anna exhibited three points in the presence of Hopkins and one point in the presence of a primate care technician. Flora exhibited two points in the presence of Hopkins. Anna and Flora were not rewarded following points to anyone other than Leavens. Clint was rewarded with food after 5 of the 14 points exhibited to people other than Leavens (the probability of reward equaled .357 in this circumstance, demonstrating less success in obtaining a food reward when generalizing to others).

\section{Points Directed to Nonfood Objects}

The females, who did not interact with the LRC-CTS, were never observed to point to the apparatus or any other nonfood item. In contrast, 27 of Clint's points were directed to nonfood items (e.g., at the computer after an incorrect response) and 120 were directed to food items (20 cases were discarded from this analysis because of ambiguity). Clint exhibited gaze alternation, vocalizations, or both significantly more often when the object of the point was food than a nonfood item, $\chi^{2}(3, N=147)=14.5, p<.01$ (see Figure 1). It may be important to note that neither of the two points emitted by Clint in the absence of a human observer was directed at food items; instead, both were directed at the computer testing apparatus immediately after an incorrect response, suggesting that the motivational basis for points to nonfood items may differ from points to food items.

The mean duration of the 27 points emitted by Clint to nonfood objects was $2.8 \mathrm{~s}(S D=2.0)$. To assess structural changes as a function of the object of Clint's points, the numbers of right- and left-handed, nonfood-directed, singlehanded points were subtracted from the total corpus of this subject's points for which handedness could be assessed. Effects of digit use were also assessed similarly. The handedness of these points did not change as a function of the object of the point, $\chi^{2}(1, N=157)=3.635, n s$, but the digit use did, $\chi^{2}(1, N=157)=9.547, p<.01$. Thus, relatively more whole-handed points were directed toward nonfood than toward food objects and indexical or single-digit points were directed more toward food than toward nonfood objects. Twenty-three of these points were directed at the computer apparatus: 18 after an incorrect response, 1 after a

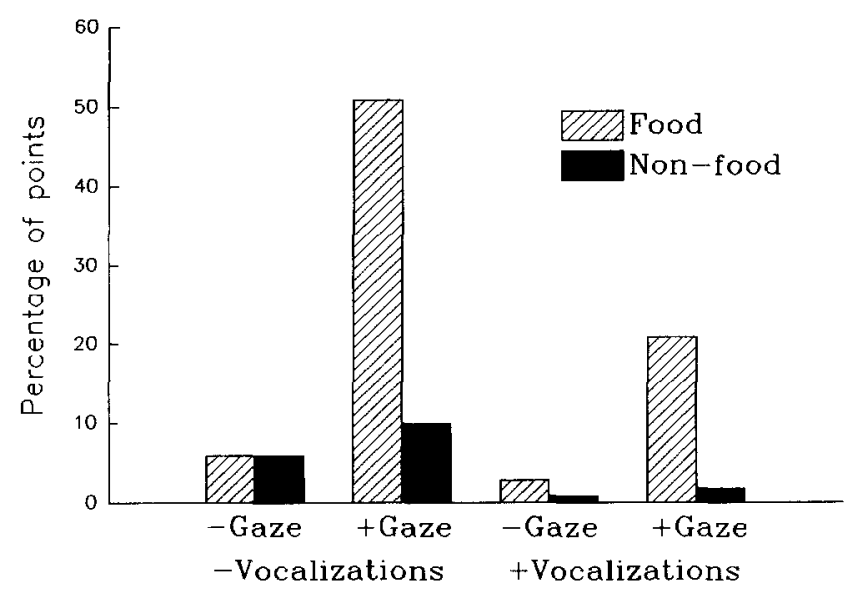

Figure 1. Significant tendency of Clint to exhibit gaze alternation where the object of the point is food, rather than a nonfood item, $\chi^{2}(3, N=147)=14.50, p=.002$. Plus signs indicate with; minus signs indicate without. 
correct response, and 4 in more ambiguous contexts (i.e., the points did not seem to be emitted in response to the audible discriminative tone signaling whether the trials were correct). One point was directed toward the reward delivery tube that Clint had knocked out of position, immediately on the return of the experimenter. Three points were directed at the experimenter's shoes, which were subsequently probed by Clint.

\section{Digit Use}

All 3 subjects exhibited significant laterality effects (Table 2). Right-handed pointing was characterized by singledigit extension, whereas left-handed pointing was typically with the whole hand, $\chi^{2}(1, N=233)=111.37, p<.001$. We eliminated 14 points from the analysis because the specific digits could not be identified, and another 9 points by Clint involved the use of two hands simultaneously (these two-handed points are analyzed below). This left a total of 233 points for this comparison. The majority of nonindexical single-digit pointing involved the use of the middle finger (or third ray).

Nine points were exhibited by Clint using both hands. These data are summarized in Table 3 . In 8 of the 9 two-handed points, Clint used the left hand for a wholehand point compared to only 4 of 9 whole-handed points with the right hand. This mirrors the interaction between hand and single-digit versus whole-hand pointing found in the data for single-hand pointing.

\section{Discussion}

Points were exhibited significantly more often in the presence, compared to the absence, of an observer. These data overwhelmingly establish the communicative nature of the pointing gestures emitted by our chimpanzee subjects. Had the gestures lacked a communicative function, then the behavior should have been random with respect to observer presence. Whereas what we call whole-hand pointing has been characterized by some human infant researchers as reaching (e.g., Leung \& Rheingold, 1981), we argue that had our subjects' whole-hand points been frustrated reaches, then the incidence of whole-hand pointing should

Table 2

Distribution of Hand and Digit Use During Single-Hand Pointing

\begin{tabular}{|c|c|c|c|c|c|c|}
\hline \multirow[b]{3}{*}{ Subject } & \multicolumn{3}{|c|}{ Left hand } & \multicolumn{3}{|c|}{ Right hand } \\
\hline & \multicolumn{2}{|c|}{ Single digit } & \multirow{2}{*}{$\begin{array}{l}\text { Whole } \\
\text { hand }\end{array}$} & \multicolumn{2}{|c|}{ Single digit } & \multirow{2}{*}{$\begin{array}{c}\text { Whole } \\
\text { hand }\end{array}$} \\
\hline & Index & Other & & Index & Other & \\
\hline Clint & 13 & 0 & 95 & 25 & 17 & 7 \\
\hline Flora & 2 & 0 & 0 & 22 & 2 & 1 \\
\hline Anna & 11 & 0 & 22 & 13 & 1 & 2 \\
\hline Total & 26 & 0 & 117 & 60 & 20 & 10 \\
\hline
\end{tabular}

Note. Indexical and other single-digit points were combined into a single category for statistical analysis; $\chi^{2}(1, N=233)=111.37$, $p<.001$. For the left hand, $n=143$; for the right hand, $n=90$.
Table 3

Points Using Both Hands Simultaneously (Clint Only)

\begin{tabular}{ccc}
\hline Right hand & Left hand & Number of instances \\
\hline Whole hand & Whole hand & 4 \\
Second digit & Whole hand & 2 \\
Third digit & Whole hand & 2 \\
Second digit & Second digit & 1 \\
\hline
\end{tabular}

have been equal in the presence or absence of humans. The observer effect reported here is congruent with that found for two pointing orangutans (Call \& Tomasello, 1994); in that study, very reduced rates of pointing occurred when a human observer was either absent or had his back turned to the orangutans.

Having established the communicative function of the pointing gestures used by our subjects, we inferred intentionality through gaze behavior and attention-getting behavior. Clint's use of gaze alternation between distal objects to which he is pointing and human observers meets the criteria for intentionality established by both human infant and ape researchers (e.g., Bard, 1990; Bates et al., 1975; Desrochers et al., 1995; Leung \& Rheingold, 1981; Plooij, 1978; Tomasello et al., 1994). Our data on Clint's gaze behavior, combined with the attention-getting behaviors exhibited by all 3 subjects, are consistent with the hypothesis that these chimpanzees recognized the necessity of capturing the attention of human observers in order to achieve desired goals (food items). Attention to the attentional status of the recipient of a communicative gesture has also been demonstrated in young chimpanzees (Tomasello et al., 1994).

Note that we combined gazes directed only at the observer with gazes directed only at the objects of the points in order to render our results more conservative-gazes directed at the observer might reasonably have been combined with gaze alternation and held to signify gazemonitoring-indeed, this is the interpretation widely put forth both in the child development literature (e.g., Bates et al., 1975) and studies of communication in apes (e.g., Gómez, 1991). Our more stringent requirement of gaze alternation between the putative objects of the points and the observers strengthens the interpretation of Clint's gaze behavior in intentional terms.

Intentional use of communicative gestures can also be assessed in terms of persistence to achieve a goal (e.g., Bates et al., 1975). All 3 subjects pointed more often in the $60-\mathrm{s}$ period following nonreinforcement than after food reinforcement. This persistence in pointing after lack of reinforcement strongly bolsters an interpretation of the pointing behavior in terms of the subjects' goals.

In addition to the measure of persistence in terms of subsequent frequency of pointing, the durations of points relative to reinforcement latencies provide additional evidence for the goal-directedness of pointing in our subjects. The points were terminated, on average, about $3 \mathrm{~s}$ after verbal acknowledgments of the gestures, but nearly $6 \mathrm{~s}$ before actual delivery of food rewards to our subjects. This implies that the discriminative stimulus signaling a success- 
ful communicative attempt (with consequent reinforcement) for the chimpanzees occurred sometime after a verbal acknowledgment and before the actual delivery of food to the animals' mouths.

In sum, the data suggest that Clint, Anna, and Flora were exhibiting intentional use of the pointing gesture. The same criteria we used (observer effects, gaze alternation, attention-getting behavior, and persistence) have also been asserted to demonstrate the attribution of agency to adults by children (e.g., Baron-Cohen, 1989, 1991); however, our data can be explained in cognitive terms without claiming that our subjects attribute mental states or agency to human observers.

Tomasello and Call (1994) suggested that pointing was an example of the uniquely human ability to understand the behavior of others in terms of the intentions that guide that behavior; in their view, only where "others are constantly attempting to direct one's attention and behavior" (Tomasello \& Call, 1994, p. 300) can apes develop this understanding. The data presented here do not require for their explanation the attribution of intentions to humans on the part of our chimpanzee subjects, though clearly the humans who respond to the chimpanzees' points attribute intentions (correctly, in our analysis) to the chimpanzees. Thus, our study dissociates pointing from the attribution of intentions by the pointing individual, with obvious implications for interpretations of the human infant pointing literature.

Following Gómez (1991), we argue that intentional communication implies knowledge that others are perceivers, that is, that intentionally communicating individuals (here, chimpanzees) are aware of the need to establish mutual attention with another social agent (here, human observers) to achieve some goal, where successful completion of that goal requires manipulation of the behavior of that social agent. We consider these data to be evidence of perspectivetaking in that it seems unlikely that the gaze alternation we observed in Clint could be parsimoniously explained without invoking the same functional explanation invoked for gaze alternation in human infant pointing (i.e., that the gaze alternation is part of a gaze-monitoring process and implies the awareness that others have a visual perspective that may differ from one's own).

It is possible that the incorporation of gaze alternation into Clint's pointing behavior represents incidental operant conditioning in the absence of any conscious or deliberate attempt by the experimenters to teach this behavior. This interpretation is consistent with the post hoc variable reinforcement schedules over the 3 subjects. We consider a purely operant interpretation of our results unlikely, however, for a number of reasons. The fallen food alone did not elicit pointing; neither did the previously fallen food in the presence of the experimenter reliably elicit pointing. Clint did not always point in the presence of a fallen food item, even when an observer was present when the item was misdelivered. If this was incidental operant conditioning, Clint would be expected to exhibit a point immediately and consistently whenever the two conditions were met, on the arrival of a human in the condition of misdelivered food items; however, although this occurred frequently, Clint often also waited several minutes after the arrival of a human observer to call the observer's attention to the fallen food. In other words, neither the misdelivery of a food reward nor the arrival of an observer (as contrasted with observer presence) was a necessary or sufficient condition for the generation of a point by Clint. Moreover, under some conditions, such as after the observer verbally and behaviorally acknowledged the point, the point was terminated although both observer and out-of-reach food continued as stimulus conditions (though, on average, points were maintained $2.56 \mathrm{~s}$ after verbal responses, over all 3 subjects).

Additionally, when the likelihood that the gesture would be understood was reduced (such as when a less familiar person walked by), a higher proportion of points was accompanied by attention-getting behavior and monitoring behavior. In other words, when the point was used with a human with whom Clint had less history in the use of the gesture, he appeared to engage in more attention-getting behavior. This flexibility in behavior seems inconsistent with a purely operant interpretation of these data, though this study cannot disconfirm such an interpretation. In short, these data are amenable to both cognitive and behaviorist interpretations; however, we would not support an interpretation that explained, for example, the pointing behavior of chimpanzees in operant terms and the pointing behavior of human 12-month-olds, or adults, in cognitive terms.

With respect to the laterality effects reported here, it should be emphasized that the accidentally misdelivered food items fell virtually always to Clint's right, and therefore the side of stimulus presentation was not controlled in this study. Nevertheless, it is worth noting that the pattern of single-digit use in pointing in this study (see Table 1), which was biased toward the right hand, resembles the same subjects' digit use in an independent measure of handedness used in our laboratory, the bimanual tube task (Hopkins, 1995). In this task, peanut butter is smeared on the inside of a short length of narrow PVC pipe and observers record which digit is used to extract the peanut butter. Clint used right-hand single digits for $92 \%$ of peanut butter extractions $(n=509)$, Anna for $100 \%(n=47)$, and Flora for $77 \%$ $(n=424)$. Given that all three of our subjects are righthanded in the bimanual tube task, the pattern toward increased dexterity in the right hand (more single-digit extensions) is consistent with previous reports of human gesture use that show that better dexterity is exhibited in the dominant hand (e.g., Haaland \& Harrington, 1989; Kimura, 1973; Vaid, Bellugi, \& Poizner, 1989). Thus, despite the lack of control over locus of stimulus presentation, our data are consistent with functional and structural patterns of finger use in humans.

When the object of Clint's point was a clearly identifiable nonfood object, his points were generally shorter in duration, associated with fewer gaze alternations or vocalizations (Figure 1), and were more often emitted with the whole hand compared with points directed to food items. Because 18 of the 27 points to nonfood items were directed at the computer after an incorrect response (including the only two points emitted during this study in the absence of a human observer), it is reasonable to suggest that whole- 
hand points to nonfood items serve a different communicative function than indexical points at food items. Perhaps whole-hand points are more declarative, and single-digit points more imperative in function.

We are not aware of reports of pointing by apes in their natural habitats: Why, then, do captive apes point? Call and Tomasello (1994) argued that, with respect to captive ape pointing, "in most cases the ape was first trained in some kind of communication with humans that involved closerange pointing, for example, human sign language or pointing to keys on a keyboard" (p. 308); further, in all cases, pointing in language-trained apes develops "spontaneously" (p. 308), without overt training. The only systematic developmental account of the acquisition of pointing in apes is that by Miles (1990) of a sign-language-trained orangutan, Chantek. She found that Chantek's pointing behavior followed a typically human sequence: exhibiting self, showing objects, giving objects, and using indicative pointing (cf. Bates et al., 1975). In humans, referential pointing usually develops in synchrony with the utterance of infants' first words; therefore, it is widely held that pointing is functionally associated with language use (e.g., Bates et al., 1975; Desrochers et al., 1995).

However, the corpus of previously existing data on pointing in apes is about evenly split between reports of pointing in language-naive apes (e.g., Boysen \& Berntson, 1989; Boysen et al., 1995; Call \& Tomasello, 1994; Povinelli et al., 1990, Woodruff \& Premack, 1979) and reports of pointing in language-trained apes (Call \& Tomasello, 1994; Fouts et al., 1982; Krause et al., 1995; Miles, 1990; Patterson, 1978; Savage-Rumbaugh, 1984, 1986). Our observations, coupled with previously published reports of pointing in language-naive apes, strongly suggest that the development of referential pointing may be independent of linguistic competence, even in humans (Petitto, 1988).

We find it difficult to conceive of a circumstance in which a wild ape is dependent on the capture and direction of another's attention to obtain an otherwise unattainable object that is distal to both of the interactants. In most imaginable circumstances, a feral ape can easily locomote to proximity with any desired food or object in its environment. The situation is dramatically different for the 12 month-old human infant, who is capable of establishing both an interest in a distal object and joint attention with an adult but is limited in locomotor capacity. It is precisely this circumstance that is encountered by captive, attentiondirecting apes, except that the barrier is not due to endogenous limitations on locomotion but to exogenous limitations (i.e., the cage mesh); thus, pointing is one solution to a specific problem requiring the use of another's agency (i.e., social tool use; Bard, 1990). The particular situation that requires pointing as a solution may only be encountered by apes in captive situations. With respect to the absence of reports of pointing by feral apes, we agree with Menzel (1973), who remarked that "one good reason that chimpanzees very seldom point manually is that they do not have to" [italics his] (p. 218), that is, a chimpanzee signals its interest in distal objects or locations with the orientation of its face and body.
Therefore we suggest, on the basis of our data and previous reports of pointing, that the following are requisite and sufficient for the development of pointing in apes: (a) a distal object of interest, (b) a barrier to obtaining that object, and (c) a history of positive reinforcement associated with a human (or humans) visibly positioned to obtain that object. These parameters are functionally analogous to the situations in which human infants begin to referentially point: (a) there is an object of interest, (b) there is a barrier to obtaining that object through immaturity in locomotor abilities, and (c) there is a history of reinforcement with older conspecifics visibly positioned to retrieve the objects of interest. This interpretation is consistent with the spontaneous nature of the development of pointing reported by others and identifies the specific contextual parameters of pointing behavior. In other words, although the structural aspects of pointing in apes can, and have been, easily shaped, pointing in apes and humans also emerges as a common solution to a problem, without explicit shaping.

Because our study was performed opportunistically on observation of pointing and is incidental to an unrelated project, generalizations of our results can only be considered tentative. Similar caveats are required by the low number of subjects in this study. In addition, we have little knowledge of their training histories. Our data do not directly address the acquisition of pointing in our subjects; the pointing was a fully developed phenomenon in all $3 \mathrm{chim}$ panzee subjects when we began the study (i.e., all 3 subjects exhibited pointing behavior from the very first videotaped session).

To summarize, contrary to several recent claims (Butterworth \& Grover, 1988; Corballis, 1991; Donald, 1991) referential pointing does occur in laboratory-reared chimpanzees in the absence of language training or explicit shaping. Furthermore, contra Povinelli and Davis (1994), referential pointing with the index finger also occurs. In accordance with criteria from both the human and ape developmental literatures, pointing in our chimpanzee subjects, like pointing in human infants about 12 months of age, is an intentionally communicative gesture.

\section{References}

Bakeman, R., \& Gottman, J. (1986). Observing interaction: An introduction to sequential analysis. New York: Cambridge University Press.

Bard, K. A. (1990). "Social tool use" by free-ranging orangutans: A Piagetian and developmental perspective on the manipulation of an animate object. In S. T. Parker \& K. R. Gibson (Eds.), "Language" and intelligence in monkeys and apes: Comparative developmental perspectives (pp. 356-378). Cambridge, England: Cambridge University Press.

Bard, K. A. (1992). Intentional behavior and intentional communication in young free-ranging orangutans. Child Development, 62, 1186-1197.

Baron-Cohen, S. (1989). Perceptual role-taking and protodeclarative pointing in autism. British Journal of Developmental Psychology, 7, 113-127.

Baron-Cohen, S. (1991). Precursors to a theory of mind: Understanding attention in others. In A. Whiten (Ed.), Natural theories 
of mind: Evolution, development and stimulation of everyday mindreading (pp. 233-251). Oxford, England: Basil Blackwell.

Bates, E., Camaioni, L., \& Volterra, V. (1975). Performatives prior to speech. Merrill-Palmer Quarterly, 21, 205-226.

Blake, J., O'Rourke, P., \& Borzellino, G. (1994). Form and function in the development of pointing and reaching gestures. Infant Behavior and Development, 17, 195-203.

Boysen, S. T., \& Berntson, G. G. (1989). Numerical competence in a chimpanzee (Pan troglodytes). Journal of Comparative Psychology, 103, 23-31.

Boysen, S. T., Berntson, G. G., Shreyer, T. A., \& Hannan, M. B. (1995). Indicating acts during counting by a chimpanzee (Pan troglodytes). Journal of Comparative Psychology, 109, 47-51.

Butterworth, G., \& Grover, L. (1988). The origins of referential communication in human infancy. In L. Weiskrantz (Ed.), Thought without language (pp. 5-24). Oxford, England: Clarendon Press.

Call, J., \& Tomasello, M. (1994). The production and comprehension of referential pointing by orangutans (Pongo pygmaeus). Journal of Comparative Psychology, 108, 307-317.

Corballis, M. C. (1991). The lopsided ape: Evolution of the generative mind. New York: Oxford University Press.

Desrochers, S., Morissette, P., \& Ricard, M. (1995). Two perspectives on pointing in infancy. In C. Moore \& P. J. Dunham (Eds.), Joint attention: Its origins and role in development (pp. 85101). Hillsdale, NJ: Erlbaum.

Donald, M. (1991). Origins of the modern mind: Three stages in the evolution of culture and cognition. Cambridge, MA: Harvard University Press.

Fogel, A., \& Hannan, T. E. (1985). Manual actions of nine- to fifteen-week-old human infants during face-to-face interaction with their mothers. Child Development, 56, 1271-1279.

Fogel, A., \& Thelen, E. (1987). Development of early expressive and communicative action: Reinterpreting the evidence from a dynamic systems perspective. Developmental Psychology, 23, 747-761.

Fouts, R. S., Hirsch, A. D., \& Fouts, D. H. (1982). Cultural transmission of a human language in a chimpanzee mother-infant relationship. In H. E. Fitzgerald, J. A. Mulins, \& P. Gage (Eds.), Child nurturance: Studies of development in primates (pp. 159193). New York: Plenum Press.

Gómez, J. C. (1991). Visual behavior as a window for reading the mind of others in primates. In A. Whiten (Ed.), Natural theories of mind: Evolution, development and stimulation of everyday mindreading (pp. 195-207). Oxford, England: Basil Blackwell.

Haaland, K. Y., \& Harrington, D. L. (1989). Hemispheric control of the initial and corrective components of aiming movements. Neuropsychologia, 27, 961-969.

Hopkins, W. D. (1995). Hand preferences for a coordinated bimanual task in 110 chimpanzees (Pan troglodytes): Crosssectional analysis. Journal of Comparative Psychology, 109, 291-297.

Kimura, D. (1973). Manual activity during speaking: I. Righthanders. Neuropsychologia, 11, 45-50.

Krause, M. A., Bodamer, M. D., \& Fouts, R. S. (1995, June). Communication of object location with the use of directional pointing in chimpanzees (Pan troglodytes). Paper presented at the Eighteenth Annual Meeting of the American Society of Primatologists, Scottsdale, AZ.

Leung, E. H. L., \& Rheingold, H. L. (1981). Development of pointing as a social gesture. Developmental Psychology, 17, 215-220.

Menzel, E. W., Jr. (1973). Leadership and communication in young chimpanzees. In E. W. Menzel, Jr. (Ed.), Symposia of the Fourth International Congress of Primatology: Vol. 1. Precultural primate behavior (pp. 192-225). Basel, Switzerland: Karger.

Miles, H. L. (1990). The cognitive foundations for reference in a signing orangutan. In S. T. Parker \& K. R. Gibson (Eds.), "Language" and intelligence in monkeys and apes: Comparative developmental perspectives (pp. 511-539). Cambridge, England: Cambridge University Press.

Patterson, F. G. (1978). Linguistic capabilities of a lowland gorilla. In F. C. C. Peng (Ed.), Sign language and language acquisition in man and ape: New dimensions in comparative pedolinguistics (pp. 161-201). Boulder, CO: Westview Press.

Petitto, L. (1988). "Language" in the prelinguistic child. In F. Kessel (Ed.), Development of language and language researchers (pp. 187-222). Hillsdale, NJ: Erlbaum.

Plooij, F. (1978). Some basic traits of language in wild chimpanzees? In A. Lock (Ed.), Action, gesture, and symbol: The emergence of language (pp. 111-132). London: Academic Press.

Povinelli, D. J., \& Davis, D. R. (1994). Differences between chimpanzees (Pan troglodytes) and humans (Homo sapiens) in the resting state of the index finger: Implications for pointing. Journal of Comparative Psychology, 108, 134-139.

Povinelli, D. J., Nelson, K. E., \& Boysen, S. T. (1990). Inferences about guessing and knowing by chimpanzees (Pan troglodytes). Journal of Comparative Psychology, 104, 203-210.

Savage-Rumbaugh, E. S. (1984). Pan paniscus and Pan troglodytes: Contrast in preverbal communicative competence. In R. L. Susman (Ed.), The pygmy chimpanzee: Evolutionary biology and behavior (pp. 395-413). New York: Plenum Press.

Savage-Rumbaugh, E. S. (1986). Ape language: From conditioned response to symbol. New York: Columbia University Press.

Tomasello, M., \& Call, J. (1994). Social cognition of monkeys and apes. Yearbook of Physical Anthropology, 37, 273-305.

Tomasello, M., Call, J., Nagell, K., Olguin, R., \& Carpenter, M. (1994). The learning and use of gestural signals by young chimpanzees: A trans-generational study. Primates, 35, 137154.

Vaid, J., Bellugi, U., \& Poizner, H. (1989). Hand dominance for signing: Clues to brain lateralization of language. Neuropsychologia, 27, 949-960.

Washburn, D. A., Hopkins, W. D., \& Rumbaugh, D. M. (1989). Video-task assessment of learning and memory in macaques (Macaca mulatta): Effects of stimulus movement upon performance. Journal of Experimental Psychology: Animal Behavior Processes, 15, 413-420.

Washburn, D. A., \& Rumbaugh, D. M. (1992). Testing primates with joystick-based automated apparatus: Lessons from the Language Research Center's Computerized Test System. Behavior Research Methods, Instruments, \& Computers, 24, 157-164.

Woodruff, G., \& Premack, D. (1979). Intentional communication in the chimpanzee: The development of deception. Cognition, 7 , $333-362$.

Received July 27,1995

Revision received February 23, 1996 Accepted February 27, 1996 